\title{
Lymphoepithelioma-like gastric carcinoma in a patient with rectal laterally spreading tumor: A case report
}

\author{
MIN CHEN $^{1 *}$, LINGDI YIN $^{2 *}$, YULING YAO $^{1}$, LEI WANG $^{1}$, GUIFANG XU $^{1}$, XIAOQI ZHANG $^{1}$, \\ YING LV ${ }^{1}$, QI SUN ${ }^{3}$, XIANGSHAN FAN ${ }^{2}$ and XIAOPING ZOU ${ }^{1}$ \\ ${ }^{1}$ Department of Gastroenterology, Nanjing Drum Tower Hospital, Nanjing University Medical School, Nanjing, \\ Jiangsu 210008; ${ }^{2}$ First School of Clinical Medicine, Nanjing Medical University, Nanjing, Jiangsu 210029; \\ ${ }^{3}$ Department of Pathology, Nanjing Drum Tower Hospital, Nanjing University Medical School, \\ Nanjing, Jiangsu 210008, P.R. China
}

Received January 2, 2015; Accepted February 1, 2016

DOI: $10.3892 / \mathrm{ol} .2016 .4268$

\begin{abstract}
Lymphoepithelioma-like gastric carcinoma (LELGC) is a rare neoplasm of the stomach that accounts for $1-4 \%$ of all gastric cancer cases. It is characterized by the presence of a lymphoid stroma with cells arranged primarily in micro alveolar, thin trabecular and primitive tubular patterns or isolated cells. In the present study, the case of a 50-year-old male patient with LELGC and rectal laterally spreading tumor is presented. Following endoscopic submucosal dissection, a diagnosis of carcinoma was reached and the patient underwent total radical gastrectomy. The postoperative pathological stage was IA T1bN0cM0 according to the Tumor-Node-Metastasis classification of gastric carcinoma, and the patient recovered well. The present case is reported to summarize the endoscopic and pathological characteristics of LELGC.
\end{abstract}

\section{Introduction}

Lymphoepithelioma-like gastric carcinoma (LELGC), initially described by Watanabe et al (1) in 1976 as gastric carcinoma with a lymphoid stroma, is a rare type of gastric carcinoma. It presents with a unique histological pattern that is characterized by poorly differentiated malignant cells along with massive lymphocyte infiltration of the background stroma (2). LELGC constitutes $1.1-4.6 \%$ of all gastric carcinoma cases (3), and is generally considered to have a more favorable prognosis compared with other types of gastric malignancy (4). However,

Correspondence to: Professor Xiaoping Zou or Dr Xiaoqi Zhang, Department of Gastroenterology, Nanjing Drum Tower Hospital, Nanjing University Medical School, 321 Zhongshan Road, Nanjing, Jiangsu 210008, P.R. China

E-mail: 13770771661@163.com

E-mail: 13770654281@163.com

*Contributed equally

Key words: lymphoepithelioma-like gastric carcinoma, laterally spreading tumor, Epstein-Barr virus, prognosis, lymphoid stroma the reason for its better prognosis has not been fully clarified and the underlying mechanism remains to be elucidated.

Lymphoepithelioma-like carcinoma (LELC) has been reported to occur at various anatomical sites, including the salivary gland, thymus, larynx, lung, esophagus, uterine cervix, urinary bladder and skin (5). As with the majority of gastric carcinomas, LELGC typically occurs in elderly people. Two histological patterns have been reported: The first, Regaud type, exhibits well-defined epithelial nests separated by broad areas of lymphocytic reaction, while the second pattern is characterized by tumor cells growing in a diffuse manner mimicking malignant lymphoma, and is known as the Schmincke type (6).

LELGC may also be categorized into two subsets: Epstein-Barr virus (EBV)-positive and microsatellite instability (MSI)-high carcinoma (7). It has been demonstrated that EBV is present in $>80 \%$ of LELGC cases, suggesting that LELGC is closely associated with EBV infection (7). The prevalence of MSI-high carcinoma in LELGC ranges from 7 to $39 \%$, with apparent geographic variability (7). LELGC also demonstrates a male predominance and a predisposition to the proximal stomach $(8,9)$. More precisely, the predominant locations of EBV-positive carcinomas are the cardia and middle portion of the stomach, while MSI-high carcinomas are more common in the gastric antrum (7).

The current study reports a case of LELGC occurring in a male patient with a rectal laterally spreading tumor (LST); to the best of our knowledge, this has not been reported previously. This report highlights this rare variant of gastric carcinoma and discusses the diagnosis and prognosis of LELGC.

\section{Case report}

A 50-year-old male patient with no specific past medical history was admitted to Nanjing Drum Tower Hospital affiliated to Nanjing University Medical School (Nanjing, Jiangsu) in September 2014, complaining of epigastric discomfort and occasional hematochezia. A colonoscopy performed at a local hospital (Xuyi People's Hospital, Xuyi, China) revealed an LST in the rectum, located $10 \mathrm{~cm}$ from the anus. A gastroscopy (Olympus GIF-XQ260; Olympus Corp., Tokyo, Japan) also 

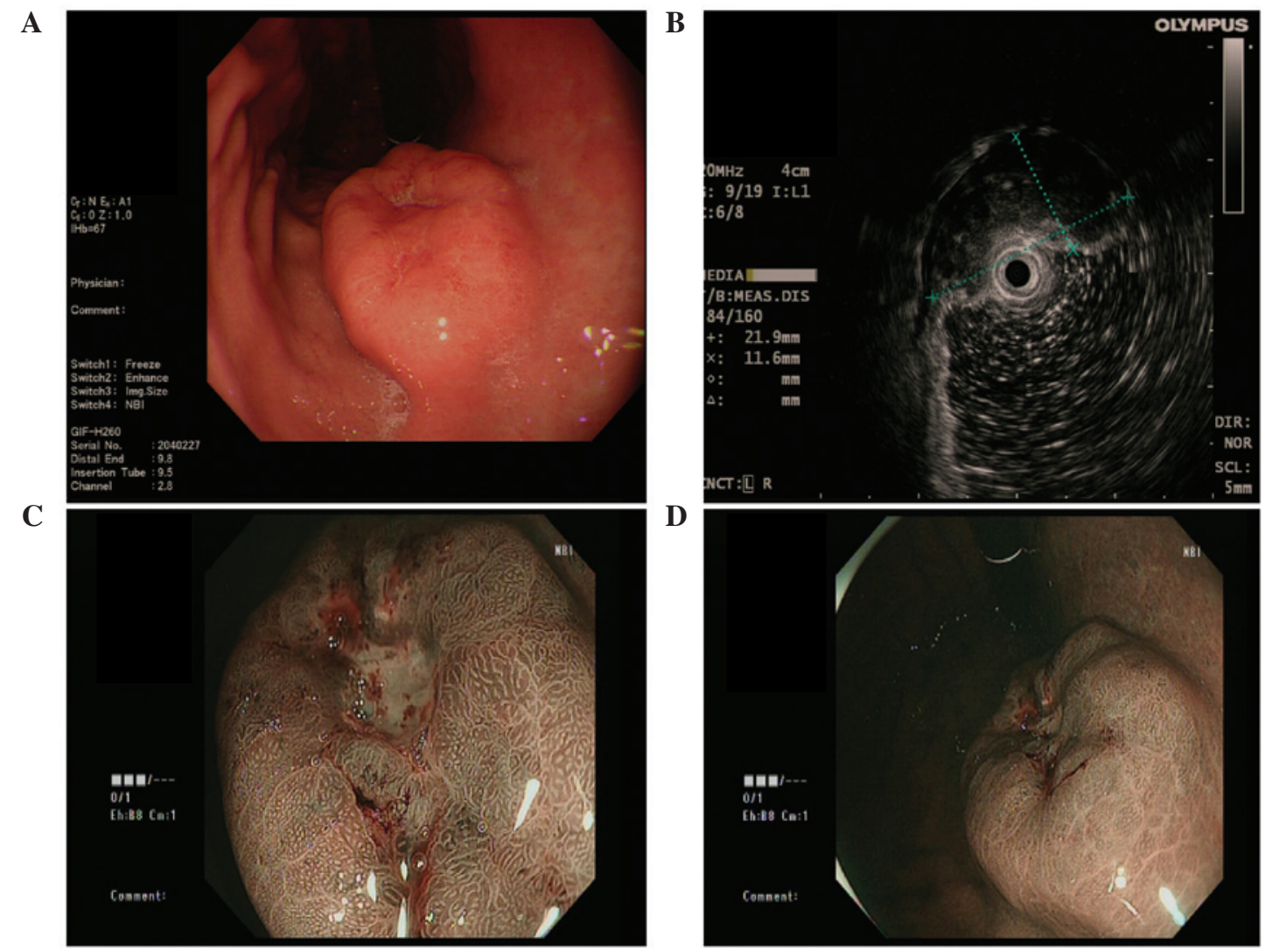

Figure 1. (A) Gastroscopy revealed a submucosal tumor-like mass covering the anterior wall of the gastric body, with a surface ulcer on top of the lesion. (B) Endoscopic ultrasound demonstrated a hypoechoic lesion originating from the submucosal layer. (C and D) Narrow band imaging of the tumor suggested no marked glandular ducts in the central area, and normal glandular ducts in the peripheral area.

revealed a submucosal columnar lesion with surface erosion at the anterior wall of the gastric body. The physical examination and laboratory tests initially performed at our hospital yielded no abnormal findings. The results were as follows: Blood, white blood cells, $6.5 \times 10^{9}$ cells $/ 1$ (normal range, $4.0-10.0 \times 10^{9}$ cells/l); neutrophils, 54.9\% (normal range, 51.0-75.0\%); urine, negative; stool/occult blood test, negative; liver and kidney functions, alanine aminotransferase, 18.9 units (U)/1 (normal range, 5.0-40.0 U/l); $\gamma$-glutamyl transferase, $17.5 \mathrm{U} / 1$ (normal range, 7.0-35.0 U/l); total bilirubin, $20.4 \mu \mathrm{mol} / 1$ (normal range, 5.0-20.5 $\mu \mathrm{mol} / \mathrm{l}$ ); direct bilirubin, $4.7 \mu \mathrm{mol} / 1$ (normal range, $1.7-6.8 \mu \mathrm{mol} / \mathrm{l}$ ); albumin, $40.0 \mathrm{~g} / \mathrm{l}$ (normal range, 35.0-51.0 g/l); blood urea nitrogen, $4.4 \mathrm{mmol} / \mathrm{l}$ (normal range, $2.9-7.5 \mathrm{mmol} / \mathrm{l}$ ); creatinine, $71 \mu \mathrm{mol} / 1$ (normal range, 44-106 $\mu \mathrm{mol} / \mathrm{l}$ ); triglyceride, $1.15 \mathrm{mmol} / \mathrm{l}$ (normal range, $0.56-1.70 \mathrm{mmol} / \mathrm{l}$ ); total cholesterol, $5.10 \mathrm{mmol} / 1$ (normal range, $2.90-5.72 \mathrm{mmol} / \mathrm{l}$ ); $\mathrm{K}^{+}, 3.77 \mathrm{mmol} / \mathrm{l}$ (normal range, 3.50-5.50 mmol/l); C-reactive protein, $2.4 \mathrm{mg} / \mathrm{l}$ (normal range, $0.0-8.0 \mathrm{mg} / \mathrm{l}$ ); blood clotting, fibrinogen, $2.1 \mathrm{~g} / \mathrm{l}$ (normal range, 2.0-4.0 g/l); blood transfusion, human immunodeficiency virus/hepatitis B/hepatitis $\mathrm{C} /$ syphilis, all negative; tumor markers, $\alpha$-fetoprotein, $2.30 \mathrm{ng} / \mathrm{ml}$ (normal range, $0.0-10.0 \mathrm{ng} / \mathrm{ml}$ ); carcinoembryonic antigen, $0.43 \mathrm{ng} / \mathrm{ml}$ (normal range, $0.0-10.0 \mathrm{ng} / \mathrm{ml}$ ); CA72-4, $0.86 \mathrm{U} / \mathrm{ml}$ (normal range, 0.0-6.9 U/ml); CA125, $1.80 \mathrm{U} / \mathrm{ml}$ (normal range, 0.0-30.2 U/ml; CA19-9, $<0.6 \mathrm{U} / \mathrm{ml}$ (normal range, 0.0-39.0 U/ml); CA242, $3.51 \mathrm{U} / \mathrm{ml}$ (normal range, 0.0-15.0 U/ml); electrocardiogram, sinus rhythm; heart rate, 68 beats/min; chest X-ray, normal.
A contrast-enhanced computed tomography (CT; Discovery CT750 HD; GE Healthcare Bio-Sciences, Pittsburgh, PA, USA) scan of the abdomen was conducted, indicating a focal thickening of the mucosa at the lesser curvature wall of the gastric body. No evidence of perigastric infiltration, enlarged lymph nodes or distant metastasis was observed by CT. Endoscopic ultrasound examination (Olympus EU-ME-1; Olympus Corp.) demonstrated a heterogeneous hypoechoic mass at the lesion site, originating from the submucosal layer and with intracavity protrusion; its transverse section measured 21.9x11.6 mm (Fig. 1). The narrow band image observation of the lesion suggested no evident glandular ducts in the central area and normal glandular ducts in the peripheral area. A biopsy indicated moderate chronic superficial gastritis with proliferation of plasma cells. Simultaneously, numerous lymphoepithelial lesions were observed. The tissues were sent to a pathologist at the Nanjing Drum Tower Hospital affiliated to Nanjing University Medical School for immunohistochemical analysis. Immunohistochemical staining confirmed the proliferative plasma cells to be of $\lambda$ type. A pathological diagnosis of lymphoma could not be established due to a lack of evidence. Endoscopic submucosal dissection (ESD) was first performed for the rectal LST (Fig. 2), and its postoperative pathology revealed villous adenoma with low-grade intraepithelial neoplasia. Subsequently. ESD of the gastric lesion was successfully performed (Fig. 3). The lesion measured $2.5 \times 2.5 \times 0.4 \mathrm{~cm}$. The specimen was obtained following the provision of consent from the patient. 
A

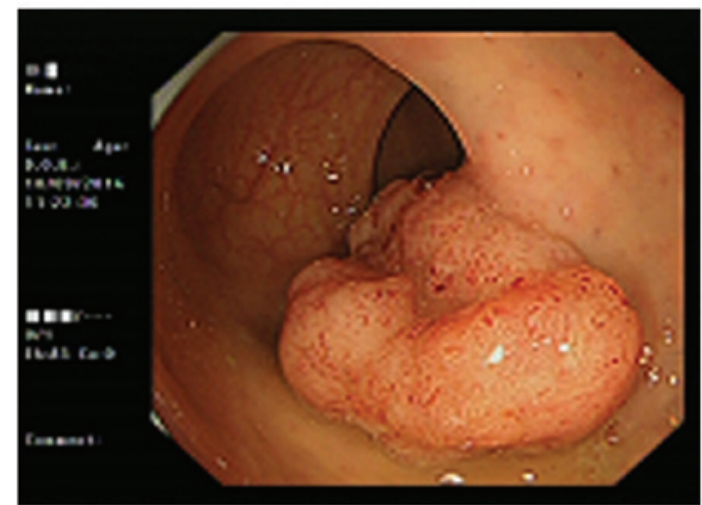

C

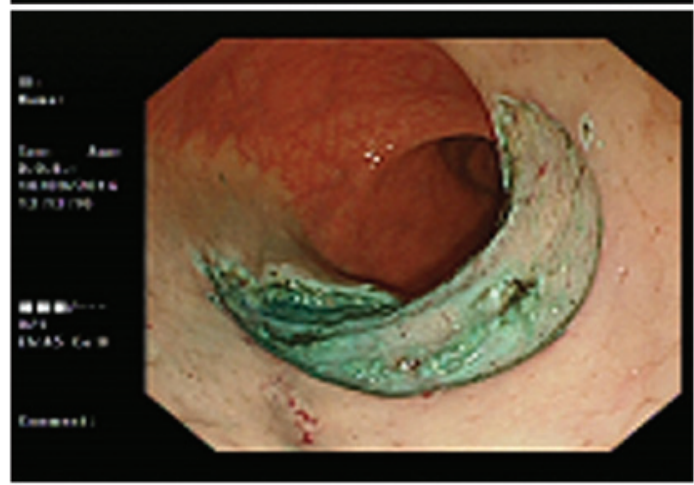

B

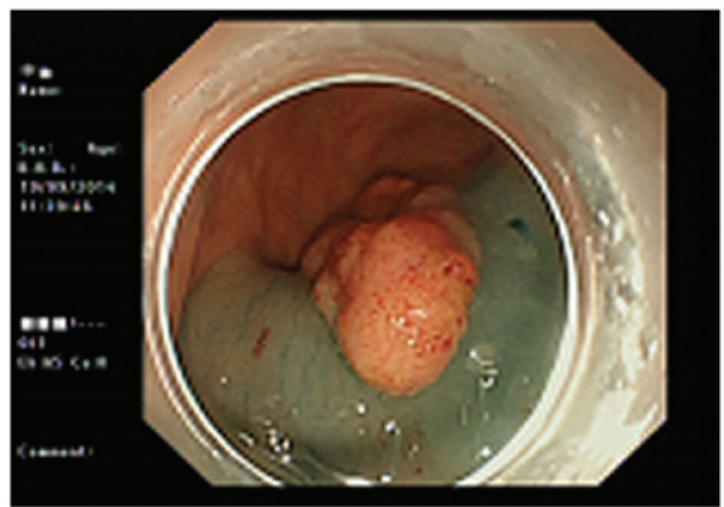

D

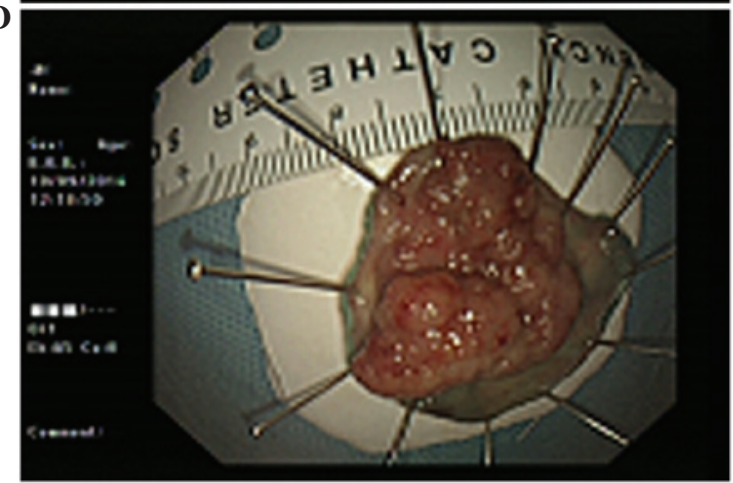

Figure 2.ESD of the rectal laterally spreading tumor was performed. (A) Endoscopic findings revealed the laterally spreading tumor in the rectum. (B) Perimeter of the lesion is marked with cautery and a lifting agent (indigo carmine) is injected into the submucosa around the perimeter of the lesion. (C) Submucosa beneath the lesion was injected and then dissected in a free-hand manner using an electrosurgical knife until the specimen was completely resected. (D) ESD specimen of the lesion in the rectum. ESD, endoscopic submucosal dissection.
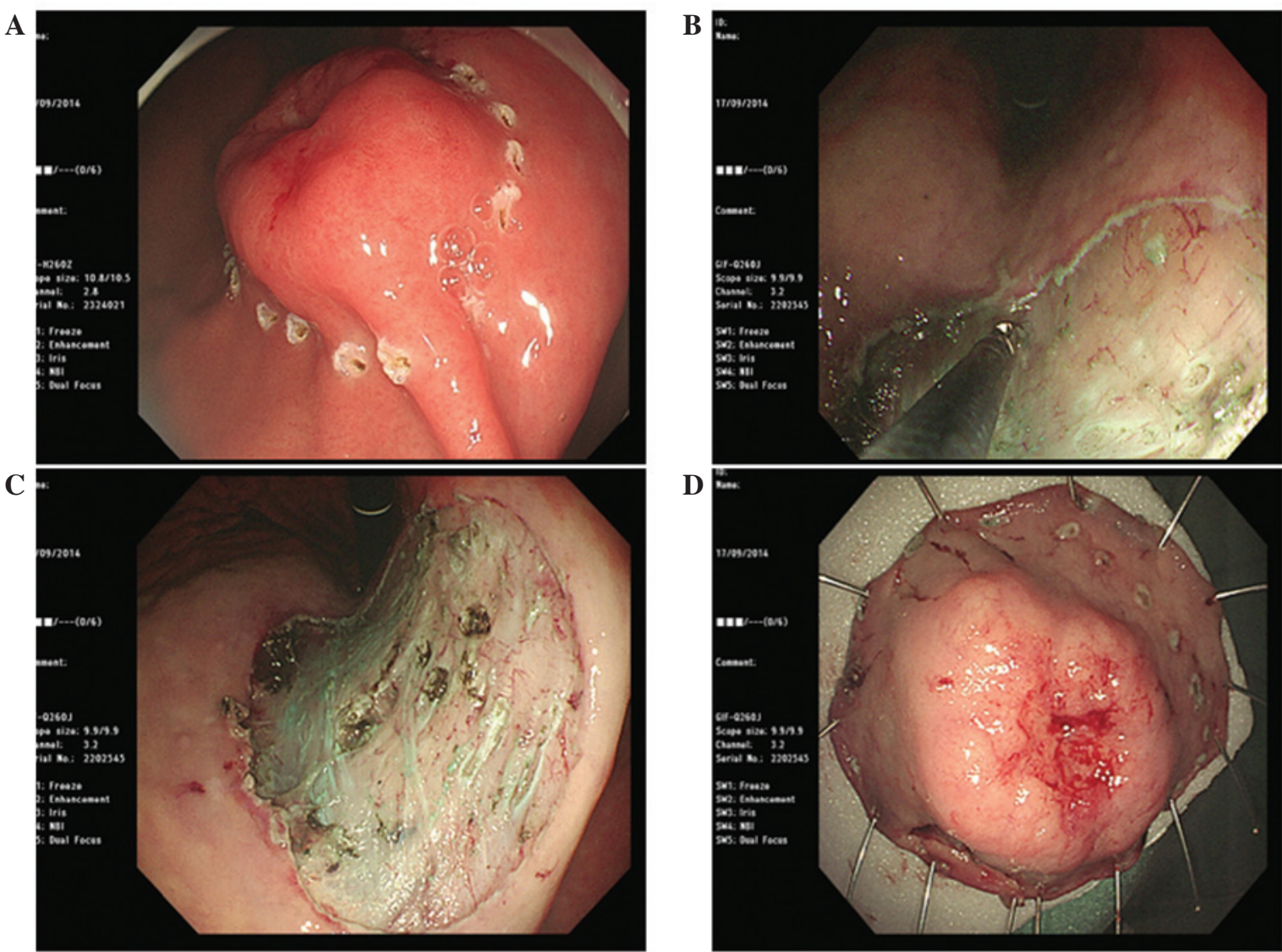

Figure 3. ESD was performed for definitive diagnosis and treatment of the gastric lesion. (A) The perimeter of the lesion was marked with a cautery knife. (B) The submucosa beneath the lesion was injected and dissected using a surgical knife. (C) The specimen in the stomach was completely resected. (D) ESD of the lesion $(2.5 \times 2.5 \times 0.4 \mathrm{~cm})$ was successful and the specimens were obtained after obtaining consent from the patient. ESD, endoscopic submucosal dissection. 
A

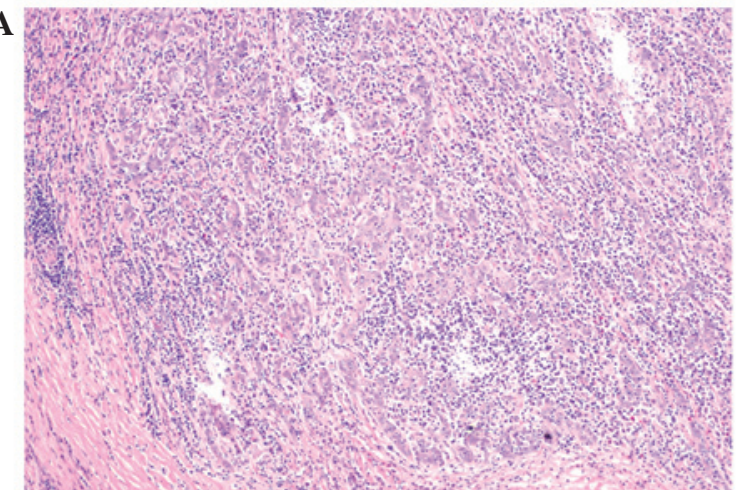

C

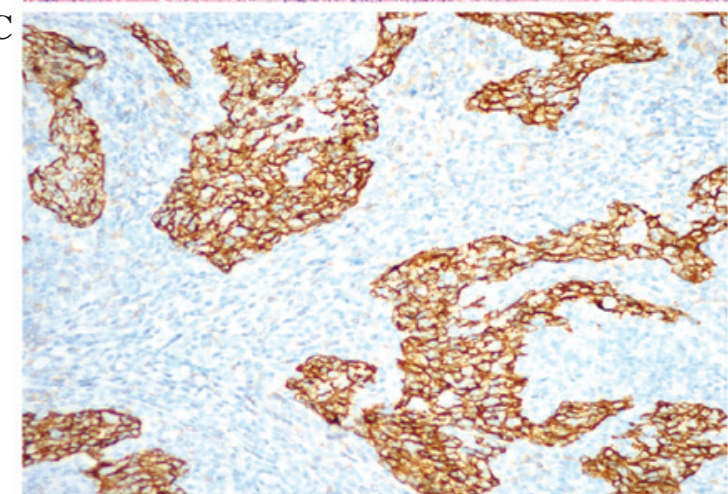

E

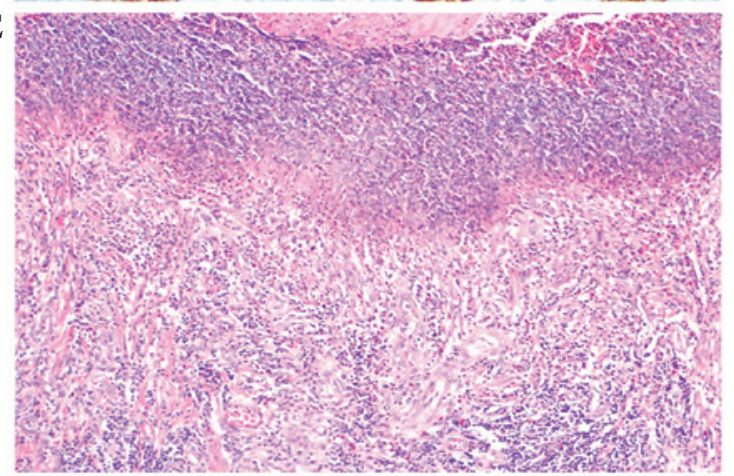

B

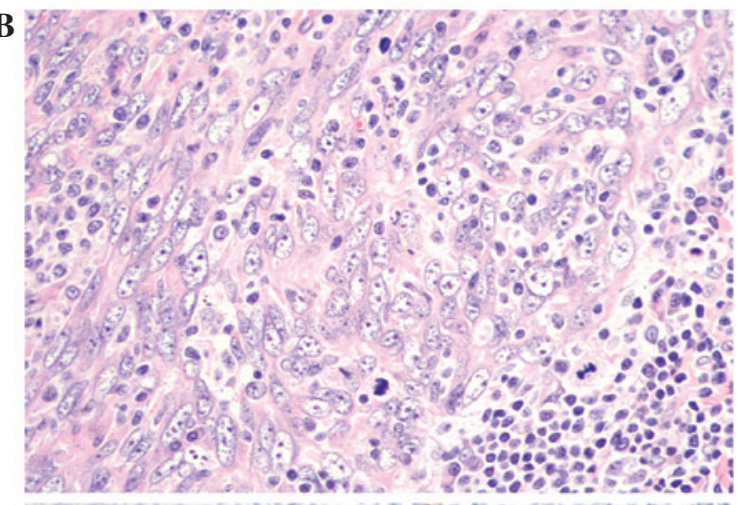

D

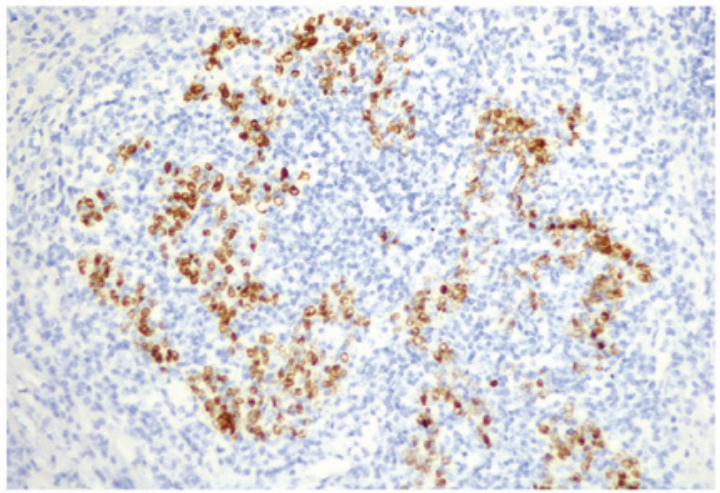

F

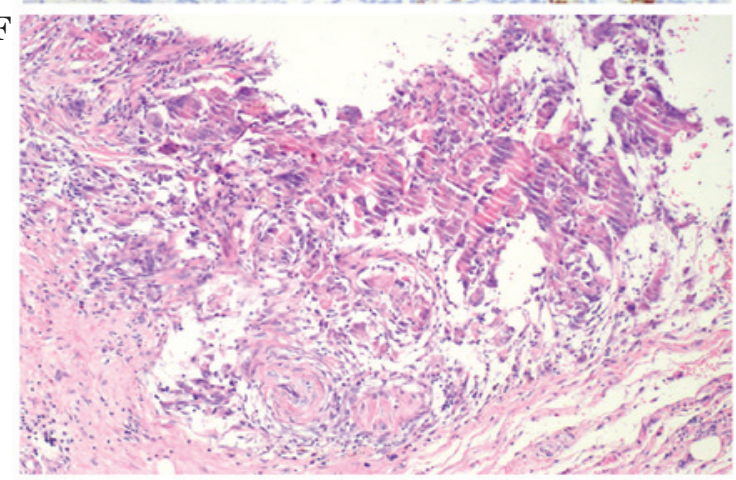

Figure 4. Pathology of ESD specimen of the gastric lesion. The histopathological and immunohistochemical examinations were compatible with LELGC. The basal margin was positive for carcinoma whereas the lateral margin was negative. The tumor had invaded the submucosal layer. (A and B) The tumor infiltrated uniformly with an abundance of lymphocytes and plasma cells throughout the entire area of the tumor. [Hematoxylin and eosin staining; (A) magnification, $\mathrm{x} 100$, (B) magnification, $\mathrm{x} 400]$. (C) Cytokeratin expression was positive in the LELGC (Envision double staining; magnification, $\mathrm{x} 200$ ) and (D) Epstein-Barr virus-encoded RNA in situ hybridization also was positive (magnification, x200). Postoperatively, lymphoepithelioma-like carcinoma of the stomach was diagnosed and staged as IA T1bNOcM0 according to the Tumor-Node-Metastasis classification of gastric carcinoma. (E) Ulceration was observed in the ESD specimen (hematoxylin and eosin staining; magnification, x100); (F) no carcinoma tissue was found in the ulcer (hematoxylin and eosin staining; magnification, x200). ESD, endoscopic submucosal dissection; LELGC, lymphoepithelioma-like gastric carcinoma.

The specimens were sent to a pathologist at the Nanjing Drum Tower Hospital affiliated to Nanjing University Medical School for histological and immunohistochemical analysis. Briefly, the gastric ESD specimen was fixed in formalin (Hubei Taikang Medical Equipment Co. Ltd., Xiaogan, China) and embedded in paraffin (Hubei Taikang Medical Equipment Co. Ltd.). The tissue sections were cut into $4-\mu \mathrm{m}$ slices. For histological examination, the sections were deparaffinized by xylene ( 2 times, 10 min each; Hubei Taikang Medical Equipment Co. Ltd.), then rehydrated in ethanol (2 changes for 5 min each, including $95 \%$ alcohol for 2 min and $70 \%$ ethanol for 2 min; Hubei Taikang Medical Equipment Co. Ltd.). The sections were briefly washed in distilled water prior to being stained with Harris hematoxylin and eosin (Hubei Taikang Medical Equipment Co. Ltd.) and mounted with xylene-based mounting medium (Hubei Taikang Medical Equipment Co. Ltd.). For immunohistochemical examination, the sections were baked at $60^{\circ} \mathrm{C}$ for $6 \mathrm{~h}$, and then dewaxed in xylene and rehydrated through a sequence of decreasing concentration of alcoholic solutions (Hubei Taikang Medical Equipment Co. Ltd.). Endogenous peroxidase activity was quenched by $3 \%$ $\mathrm{H}_{2} \mathrm{O}_{2}$ (Nanjing Chemical Reagent Co., Ltd., Nanjing, China) incubation for $10 \mathrm{~min}$ at room temperature. For antigen retrieval, tissue slides were boiled in $1 \mathrm{mmol} / \mathrm{l}$ ethylenediaminetetraacetic acid ( $\mathrm{pH}$ 8.0; Nanjing Chemical Reagent Co., Ltd.) in an autoclave (catalog no., ALP-CL-32L; ALP Co., Ltd., Tokyo, Japan) for $10 \mathrm{~min}$. Subsequent to washing three times for 5 min with PBS, the sections were incubated with mouse anti-human anti-cytokeratin monoclonal antibody (dilution, 1:100; catalog no., C2562; Sigma-Aldrich, St. Louis, MO, 
USA), at $4^{\circ} \mathrm{C}$ overnight. Secondary antibody, an anti-mouse IgG antibody from the PV-9000 kit (ZSGB-BIO, Beijing, China) was diluted to 1:1000 using Tris-buffered saline with Tween 20 (100 ml) containing skim milk (5 g; Brightdairy Co. Ltd, Shanghai, China). The slices were then incubated with the secondary for $30 \mathrm{~min}$ at room temperature susequent to washing three times with PBS (Nanjing Chemical Reagent Co., Ltd.). The immunoreactivity was revealed using diaminobenzidine (Wuhan Boster Biological Technology, Ltd., Wuhan, China) as the final chromogen. Finally, the sections were counterstained with hematoxylin and then dehydrated and mounted. Negative controls were carried out by omission of the primary antibody. Known immunostaining positive slides were used as positive controls. All slides were observed using an Olympus BX53 microscope (Olympus Corporation, Tokyo, Japan).

Histopathological and immunohistochemical examinations of the gastric lesion ESD specimen were compatible with LELGC (Fig. 4) (7). The cytokeratin (pan) staining revealed the existence of tumor cells that were derived from epithelial tissue, and in situ hybridization (ISH) was used to confirm the presence of EBV. EBV-encoded small RNA (EBER) is abundant in each latently infected cell (up to $10^{7}$ molecules/cell). Using EBER ISH (StatSpin ThermoBrite; Abbott Laboratories, Abbott Park, IL, USA), all or the vast majority of neoplastic cells show positive signals in positive cases. EBER-ISH has been used as a gold standard to identify EBV-associated gastric carcinomas. Furthermore, the basal margin was positive for carcinoma, whereas the lateral margin was negative. The tumor had invaded the submucosal layer. Therefore, on the 7th day after ESD, the patient received an additional total radical gastrectomy. Dissection of group 1-9 lymph nodes was completed. No local metastases, enlarged lymph nodes or carcinoma-like tissues were observed in the stomach. The postoperative pathological stage was IA T1bN0cM0 according to the Tumor-Node-Metastasis classification of gastric carcinoma (10). At a follow-up 3 months subsequent to the ESD, the patient was found to have recovered well from surgery, with no recurrence. The patient has received no further treatment, and at the most recent follow-up appointment in September 2015 no positive findings were observed following gastroscopy and CT.

\section{Discussion}

LELGC is a type of gastric carcinoma with characteristic clinicopathologic features $(6,11)$; it is also called gastric carcinoma with lymphoid stroma (9). Following the publication of the report by Moore and Foote (12) indicating that medullary carcinoma of the breast with lymphoid infiltration is associated with EBV infection, similar findings were reported regarding carcinomas of the stomach (1). According to the World Health Organization classification of tumors of the digestive system, LELGC is a type of tubular carcinoma (13). It has been reported that lymphocytic infiltration in LELGC may be a host defensive reaction against the cancer, and that a greater extent of lymphocytic infiltration may be associated with better prognosis (4).

Patients with LELGCs frequently exhibit no significant symptoms, and when clinical symptoms are exhibited they are often similar to conventional gastric adenocarcinoma (8).
Furthermore, LELGCs are often macroscopically mistaken for submucosal tumors (SMTs) (6). Therefore, accurate diagnosis may be difficult prior to surgery, even if an endoscopic biopsy is performed. Lesions composed of lymphocytic infiltration may be mistaken for an intense reactive lymphoid infiltrate or even lymphoma (5). In fact, in the present case, a definitive diagnosis of LELGC could not be established using the sample obtained by endoscopic biopsy; despite performing the endoscopic biopsy twice, a definitive diagnosis could not be determined as the tumor mimicked SMT. Subsequently, ESD for local resection was conducted, and a diagnosis of LELC was established on the basis of the histological characteristics of the dissected specimen. Takahashi et al (14) previously demonstrated a strategy for the diagnosis and treatment of gastric SMTs by laparoscopic surgery based on tumor measuring, and reported that this procedure may be useful as it aids accurate diagnosis and may be followed by curative surgery. Therefore, we speculate that laparoscopic surgery could be replaced by ESD, as was conducted in the present case. Lee et al (6) reported that ESD treatment of EBV-associated early LELC may have favorable long-term outcomes, despite deep submucosal invasion of tumor cells. Therefore, a conservative management strategy without additional surgery may be considered for EBV-positive early LELC with submucosal invasion treated by ESD, particularly in patients with severe comorbidity or high surgical risk (6). To enable conservative management, intensive medical follow-up must be performed using esophagogastroduodenoscopy (EGD) and abdominal CT scans (4).

As mentioned, LELGC has been reported to have a favorable prognosis compared with ordinary gastric carcinoma (4). Specifically, EBV-positive as well as MSI-high tumors in general have themselves been variably associated with a survival advantage (4). Whether the advantage is related to the lymphoid infiltrate, or whether the EBV or MSI statuses themselves serve as independent prognosticators remains to be explained (7). However, large-scale research by Beghelli et al (15) concluded that the MSI phenotype (stage II only) is significantly associated with survival.

In summary, the current study describes a case of LELGC in a patient with rectal LST. LELGC is a rare morphological variant of gastric carcinoma that has special clinical and histological features that distinguish it from ordinary gastric adenocarcinomas. The endoscopic findings revealed a submucosal columnar lesion with surface erosion at the anterior wall of the gastric body. The diagnosis of LELGC should primarily rely on the characteristic morphology and detection of EBV by EBV-encoded RNA, polymerase chain reaction (PCR), or Southern blot technique, or detection of MSI-high status by immunohistochemistry for DNA repair proteins and/or microsatellite PCR of specific markers. It is recommended that screening using EGD is necessary for patients with symptoms relating to the digestive system, including hematochezia. Understanding the clinical and histopathological features of LELGC is important in the preoperative diagnosis and in differentiating this entity from other tumors, which has decisive effect on the selection of treatment and the final outcome. The present study describes a classical case of LELGC with positive EBV and cytokeratin expression according to in situ hybridization and immunohistochemical analysis. 


\section{Acknowledgements}

The authors would like to thank Dr Xiangshan Fan and Dr Qi Sun (Department of Pathology, Nanjing Drum Tower Hospital, Nanjing, University Medical, School, Nanjing, China) for their pathological guidance and diagnosis.

\section{References}

1. Watanabe H, Enjoji M and Imai T: Gastric carcinoma with lymphoid stroma. Its morphologic characteristic and prognostic correlations. Cancer 38: 232-243, 1976.

2. Gromski MA, Miller CA, Lee SH, Lee TH, Chung IK, Park SH, Kim SJ and Cho HD: Gastric lymphoepithelioma-like carcinoma mimicking a subepithelial lesion treated by endoscopic submucosal dissection. Gastrointest Endosc 76: 419-421, 2012.

3. Shibata D, Tokunaga M, Uemura Y, Sato E, Tanaka S and Weiss LM: Association of Epstein-Barr virus with undifferentiated gastric carcinomas with intense lymphoid infiltration. Lymphoepithelioma-like carcinoma. Am J Pathol 139: 469-474, 1991.

4. van Beek J, zur Hausen A, Klein Kranenbarg E, van de Velde CJ, Middledorp JM, van den Brule AJ, Meijer CJ and Bloemena E: EBV-positive gastric adenocarcinomas: A distinct clinicopathologic entity with a low frequency of lymph node involvement. J Clin Oncol 22: 664-670, 2004.

5. Shibata D and Weiss LM: Epstein-Barr virus-associated gastric adenocarcinoma. Am J Pathol 140: 769-794, 1992.

6. Lee JY, Kim KM, Min BH, Lee JH, Rhee PL and Kim JJ: Epstein-Barr virus-associated lymphoepithelioma-like early gastric carcinomas and endoscopic submucosal dissection: Case series. World J Gastroenterol 20: 1365-1370, 2014.

7. Grogg KL, Lohse CM, Pankratz VS, Halling KC and Smyrk TC: Lymphocyte-rich gastric cancer: Associations with Epstein-Barr virus, microsatellite instability, histology and survival. Mod Pathol 16: 641-651, 2003.
8. Song HJ, Srivastava A, Lee J, Kim YS, Kim KM, Ki Kang W, Kim M, Kim S, Park CK and Kim S: Host inflammatory resoponse predicts survival of patients with Epstein-Barr virus-associated gastric carcinoma. Gastroenterology 139: 84-92, 2010.

9. Herath $\mathrm{CH}$ and Chetty R: Epstein-Barr virus-associated lymphoepithelioma-like gastric carcinoma. Arch Pathol Lab Med 132: 706-709, 2008.

10. Ajani J, D'Amico TA, Hayman JA, Meropol NJ and Minsky B; National Comprehensive Cancer Network: Gastric cancer. Clinical practice guidelines in oncology. J Natl Compr Canc Netw 1: 28-39, 2003.

11. Cheng N, Hui DY, Liu Y, Zhang NN, Jiang Y, Han J, Li HG, Ding YG, Du H, Chen JN and Shao CK: Is gastric lymphoepithelioma-like carcinoma as a special subtype of EBV-associated gastric carcinoma? New insight based on clinicopathological features and EBV genoma polymorphisms. Gastric Cancer 18: 246-255, 2015.

12. Moore OS Jr and Foote FW Jr: The relatively favorable prognosis of medullary carcinoma of the breast. Cancer 2: 635-642, 1949.

13. Fenoglio-Preiser C, Carneiro F, Correa P, Guilford P, Lambert R and Megraud F: Gastric carcinoma. In: World Health Organization Classification of Tumours - Pathology and Genetics of Tumours of the Digestive System. Hamilton SR and Aaltonen LA (eds). IARC Press, Lyon, France, pp37-52, 2000.

14. Takahashi T, Otani Y, Yoshida M, Furukawa T, Kameyama K, Akiba Y, Saikawa Y, Kubota T, Kumai K, Kuramochi S, et al: Gastric cancer mimicking a submucosal tumor diagnosed by laparoscopic excision biopsy. J Laparoendosc Adv Surg Tech A 15: 51-56, 2005.

15. Beghelli S, de Manzoni G, Barbi S, Tomezzoli A, Roviello F, Di Gregorio C, Vindigni C, Bortesi L, Parisi A, Saragoni L, et al: Microsatellite instability in gastric cancer is associated with better prognosis in only stage II cancers. Surgery 139: 347-356, 2006. 